\title{
Experimental Study on Bubble Dynamics and Wall Heat Transfer Arising from a Single Nucleation Site at Subcooled Flow Boiling Conditions - Part 1: Experimental Methods and Data Quality Verification
}

\author{
Junsoo Yoo ${ }^{\mathrm{a}, *}$, Carlos E. Estrada-Perez ${ }^{\mathrm{b}}$, Yassin A. Hassan ${ }^{\mathrm{a}, \mathrm{b}}$ \\ ${ }^{a}$ Department of Nuclear Engineering, Texas A\&M University, 253 Bizzell Hall West, 3133 TAMU, College Station, Texas 77843- \\ 3133, USA \\ ${ }^{b}$ Department of Mechanical Engineering, Texas A\&M University, 100 MEOB, 3123 TAMU, College Station, Texas 77843-3123, \\ USA \\ *Corresponding author: Tel. +1 979-845-4161; Email address: kaks2000@gmail.com (J. Yoo)
}

\begin{abstract}
A novel experimental method and data acquisition/analysis strategy that ensures reliable measurements of various subcooled flow boiling parameters is discussed. In this study, all experiments were performed by keeping a single active nucleation site within the entire heated area in a square upward flow channel. This approach greatly facilitated the observation of bubble and wall heat transfer features in subcooled boiling flow.

Vapor bubbles originating from the nucleation site were observed with both micro- and macroscopic views from high-speed cameras while corresponding wall temperature was measured by an infrared camera. This allowed simultaneously capturing various bubbles characteristics with multiple scales (both space and time) as well as their impact on wall heat transfer. In addition, efforts were made to characterize the observed boiling behavior with high statistical accuracy by analyzing numerous images taken at each test condition.

This study proves that by taking the current strategy excellent repeatability and thus reliability can be achieved for a wide range of flow boiling parameters such as bubble size, bubble velocity, statistical distribution of bubble size and time-averaged wall heat transfer coefficients. Also, the major sources of uncertainty for each measurement are thoroughly investigated, from which the final uncertainties are determined. Overall, the present study suggests what we must concern to achieve truly reliable and useful data from any boiling experiments.
\end{abstract}


Key words: Subcooled boiling flow, single nucleation site, reliability, statistics

\section{Introduction}

Forced convective boiling has been extensively studied both theoretically and experimentally over the past decades because of its practical importance in the safety, reliability, and performance of many engineering applications such as nuclear reactors and electronic devices. Consequently, many empirical correlations and mechanistic models have been proposed to predict the related phenomena, which have been widely utilized in CFD simulations of two-phase flow boiling systems (Anglart et al., 1997; Bae et al., 2010; Krepper and Rzehak, 2011; Podowski and Podowski, 2009; Yeoh et al., 2008). However, the ability to predict the complex thermal-hydraulic processes in boiling systems using CFD with such constitutive models/correlations still remains unsatisfactory. One of the key issues here is that the level of confidence of CFD prediction employing multi-fluid approach strongly relies on the validity of constituent closure models. Therefore, to overcome the current situation, the limitations of existing closure models should be clearly understood and improved based on the correct physical understanding of the complex processes associated with boiling. To this end, reliable experimental data that can be used for model validation and development are of critical importance. However, producing such high-quality data from any boiling experiment is not a simple task, and this difficulty is one of the major reasons why we can often find substantial scattering and/or inconsistency of the measured bubble parameters such as departure/lift-off bubble size and bubble frequency in the literature (Chu et al., 2011; Prodanovic et al., 2002; Situ et al., 2005; Situ et al., 2008). The major issues and/or difficulties affecting measurement accuracy in boiling experiments can be summarized as follows:

(1) The difficulty of measuring and characterizing boiling process at a given condition originates from the chaotic nature of the phenomenon. That is, the experimental results of boiling parameters such as bubble size and frequency can be considerably affected even by small changes of the test boundary conditions. Also, boiling can often be initiated unexpectedly due to small perturbations such as the random deposition of small impurities on the heater surface; and such incipience of boiling usually affects the overall heat transfer characteristics of the system significantly while increasing its phenomenological complexity. (2) Another issue results from the inherently stochastic nature of the boiling parameters. For instance, the bubble size and frequency measured under a given boiling condition are always presented as a distribution rather than a single deterministic value. This implies that such distributions are important for the 
description of boiling phenomena and should be well-characterized in addition to the statistical average of the measured parameters. Also, the stochastic nature of boiling parameters usually requires the analysis of numerous samples in order to correctly represent the boiling characteristics at a given test condition.

(3) As revealed in our previous study (Yoo et al., 2014), due to the local, fast, and random nature of the wall nucleation process, the observation view of nucleating bubbles and/or camera resolution can cause significant mis-measurement which leads to inaccurate experimental results of boiling parameters.

(4) Finally, gaining complete insight into the boiling heat transfer mechanism based on the limited number of parameters measured in a single experiment is always challenging because the boiling process generally accompanies a variety of thermal-hydraulic sub-processes which are tightly coupled to one another.

To the best of our knowledge, despite the extensive experimental works reported in literature, especially for forced convective boiling (Abdelmessih et al., 1972; Chu et al., 2011; Klausner et al., 1993; Li et al., 2013; Okawa et al., 2005; Unal, 1976), few works are free of those issues mentioned above. This is often due to the limitations of available experimental techniques and/or the fact that addressing such issues in boiling experiments has yet to be well recognized by experimentalists. Of course, our understanding on the boiling phenomena has progressed substantially due to such previous experimental efforts; and a number of empirical correlations (Ivey, 1967; Kocamustafaogullari and Ishii, 1983) and mechanistic models (Klausner et al., 1993; Yeoh et al., 2008; Zeng et al., 1993) have been developed and validated based on them. However, in order to fill the gap that is still debatable regarding the convective boiling heat transfer mechanism and its modeling, a more precise experimental approach is required. In particular, the effort to overcome the shortcomings existing in earlier experimental works related to the measurement issues described in the list above can be a good start to reach the goal more efficiently.

In this context, we devised a subcooled flow boiling experiment in a square, vertical, upward flow channel. The main objective is to improve the fundamental insight into the subcooled flow boiling process through the intensive and extensive observation of boiling parameters with a multi-scale observation approach. For this, highspeed photography and infrared (IR) thermometry were simultaneously employed based on the experimental method established by Yoo et al. (2015). Using this method, we could capture both bubble dynamics and boiling heat transfer features with high fidelity in a single flow boiling facility. In addition, a special effort was made to better investigate the characteristics of thermal-hydraulic process within the subcooled flow boiling channel by addressing the anticipated issues of boiling measurement as follows: (i) To avoid the phenomenological complexity caused by 
the random presence of nucleation sites, the heater was specially designed to keep a single active nucleation site over the entire heated area during the experiments. (ii) To improve the reliability of experimental results, a large number of images were taken and analyzed for each test condition; and the discussion was made after properly characterizing the observed behaviors rather than discussing them after taking a few samples at a selected moment. The number of images (or amount of data) used to characterize the bubble behaviors was usually much more than those used in the statistical analysis of bubble characteristics reported in literature (Klausner et al., 1993; Maurus and Sattelmayer, 2006; Thorncroft et al., 1998). (iii) To investigate the various aspects of bubble behaviors within the test channel, the bubble behaviors were recorded using simultaneously three high-speed cameras at different resolutions (i.e., multi-scale observation). (iv) To gain deeper insight into the complex flow boiling phenomena, various parameters such as bubble size, bubble velocity, bubble release frequency, bubble size distribution, and wall heat transfer coefficients were investigated together, and relevant relations among them were studied. (v) Lastly, the physical parameters of interest were observed within the relatively large area of the flow path rather than at a local position (e.g., a nucleation site), enabling investigation of the axial development of such parameters as well.

This paper, the first in a series describing our work with subcooled flow boiling experiment, focuses on discussing the novel experimental methods which assure reliable measurement of various flow boiling parameters and thus allow better insight into the heat transfer mechanism induced by vapor bubbles. In the following sections, the experimental setup, optical measurement strategy, image acquisition/analysis method, and data quality achieved from the present work are described in detail.

\section{Experimental facility and measurement strategy}

\subsection{Flow boiling loop}

The flow boiling loop was designed to perform the subcooled flow boiling experiment in a vertical square test section at atmospheric pressure. The refrigerant HFE-301 (Novec ${ }^{\mathrm{TM}}$ 7000, 3M Inc.) was employed as working fluid (boiling point: $34{ }^{\circ} \mathrm{C}$ at $1 \mathrm{~atm}$ ). As shown in Figure 1, the main components of the loop included a centrifugal pump, control valves, a heat exchanger, a test section, and a degassing tank. The working fluid was circulated through the loop by the centrifugal pump which delivered a constant volumetric flow; the flow rate was manually controlled for the experiments by means of a control valve installed downstream of the pump and upstream of the test section. The heat exchanger was used to control the fluid temperature to the test section. At the highest position 
of the loop (downstream of the test section), a degassing tank was installed to keep the system at atmospheric pressure and to function as a phase separator. Also, to ensure hydro-dynamically fully developed flow within the test section, the flow loop was designed to include an unheated flow conditioning with the length $l / \mathrm{D}_{\mathrm{h}} \approx 61$ before the fluid enters the test section ( $l$ is the unheated channel length prior to the test section and $D_{h}$ is the hydraulic diameter of the square channel).

The test section was vertically aligned, and its flow area had a square geometry of $10 \times 10 \mathrm{~mm}^{2}$. The walls of the test section were made of transparent acrylic on three sides with the transparent heater wall serving as the fourth side. The heater wall consisted of multiple transparent layers, in which a thin layer of indium-tin-oxide (ITO) film was used as a heating element to induce the boiling. This test section design allowed us to clearly observe the bubble motions from different directions simultaneously, as shown by the camera positions in Figure 2. More details of the multi-layer heater wall design as well as the experimental strategy are discussed in section 2.2. The total height of test section was $305 \mathrm{~mm}$, and the heated length $\mathrm{L}_{0}$ was $224 \mathrm{~mm}$ (see Figure 1). The remaining part of the heater wall was painted with conductive silver paint (Ted Pella, Inc.) on both ends. These ends were subsequently connected to a DC power supply (AMETEK ${ }^{\circledR}$ Sorensen XHR 600-1.7) to provide the Joule heating through the ITO film during the experiments. Also, in order to prevent unexpected boiling caused by the fluid trapped at the joints of the side walls, the width of ITO heating film on the glass substrate was designed to be narrower $(7.5 \mathrm{~mm})$ than the square channel width of $10 \mathrm{~mm}$.

The flow loop was covered with insulating material except for the transparent test section. The heater wall on one side of test section was designed as multiple layers to minimize the possible heat loss to the environment (section 2.2). During this study, the heat loss was estimated about 4-11\%, which depended on experimental condition. The amount of heat loss was evaluated by the difference between the total heat provided by DC power supply and the heat that was actually delivered to the fluid in the test section. The latter can be estimated by the algorithm described in Yoo et al. (2015).

Prior to the experiments, the loop was degassed by heating the fluid to the saturation temperature $\left(=34^{\circ} \mathrm{C}\right)$ and continuously circulating the fluid through the loop for several hours. During the experiments, the liquid flow rate was measured using a Coriolis flow meter (Micro Motion ${ }^{\circledR}$ ELITE $^{\circledR}$ ). The fluid temperatures at the inlet and outlet of the test section were measured with T-type thermocouples (OMEGA® HYP-0 mini-hypodermic probe). 
The ambient temperature around the test section was measured using a K-type thermocouple (OMEGA HSTC series). The measurement of the ambient temperature was subsequently used to estimate the actual heating film (i.e., ITO film) temperature using IR thermometry (Yoo et al., 2015). The data obtained by each instrument were recorded (24,000 samples in 4 minutes for each test case) by the data acquisition system (National Instrument Inc.), and the average values were taken to represent the test boundary conditions during this study.

\subsection{Optical measurement strategy for multi-parameter and multi-scale observation}

An experimental approach for both enhanced two-phase flow visualization and accurate wall temperature measurement by Yoo et al. (2015) was employed and extended for the present study. Specifically, as shown in Figure 2, three high-speed cameras were arranged around the transparent test section for the micro- and macroscopic observation of bubble motions. High-speed camera (HSC) 1 was used to capture the boiling motions near the nucleation site from the side of the bubbles (side view). HSC 1 arrangement deviated about $25 \sim 30^{\circ}$ from the heater plane to reduce the optical distortions. HSC 1 had a resolution of $10 \mathrm{kHz}$ and $9.09 \mu \mathrm{m} /$ pixel. The bubble motions downstream (i.e., far from the nucleation site) were observed using the high-speed camera labeled "HSC 2". HSC 2 shared a similar arrangement with HSC 1 (i.e., side view). HSC 3 was employed to observe the bubble motions from the top of the bubbles (i.e., top view, with the heater wall defined as the bottom). HSC 3 provided a different perspective than the other two cameras over a larger area of flow path. Despite the relatively limited resolution of HSC $3(0.5 \mathrm{kHz}, 78.7 \mu \mathrm{m} / \mathrm{pixel})$, the larger view provided us with useful information that cannot be captured by HSC 1 such as sliding bubble behavior far from the nucleation site (see Figures 2 and 3). Consequently, with the cameras setup shown in Figure 2, we can observe the local and fast bubble motions (small scale) near the nucleation site as well as the larger scale bubble motions through the heater length downstream of the nucleation site (i.e., multi-scale observation). Moreover, different bubbles characteristics can be observed simultaneously by analyzing the bubble images captured by each HSC (i.e., multi-parameter observation). Details of the multi-parameter observation will be discussed in section 2.4.

In Figure 3, representative bubble images taken from the three HSCs by applying the current strategy are shown. In Figures 2 and 3, L denotes the relative axial location from the beginning of heating within $\mathrm{L}_{0}$ (i.e., $0 \leq \mathrm{L} \leq \mathrm{L}_{0}$ ); the definition $\mathrm{L} / \mathrm{L}_{0}$ is consistently used in this paper to represent the axial location of bubbles. In Figure 3, we can see the horizontal line existing at about $\mathrm{L} / \mathrm{L}_{0} \approx 0.45-0.5$ in the image taken by HSC 3 . This is because the 
sapphire window at the outer surface of heater wall consisted of two small pieces (see Figure 4 below for the heater wall design). That is, the horizontal line is due to the contact between the two sapphire windows.

During this study, the heater wall surface temperature was also measured over the entire heated area $\left(7.5 \times 224 \mathrm{~mm}^{2}\right)$ using IR thermometry. The wall temperature measurements were performed while capturing the bubble motion by the HSCs (see Figure 2). This allowed us to observe the wall heat transfer modification induced by the bubbles within the test section at different experimental conditions. All of the three HSCs and the IR camera were set up to be triggered at the same time via a BNC cable. The resolution of each camera and the total axial length observed are given in Figure 2.

Figure 4 illustrates the multi-layer heater wall design as well as the IR camera arrangement to capture the temperature of the heater surface touching the fluid (hereafter, wall). The calibration algorithm to obtain the accurate ITO film temperature through the multi-layer wall and the required experimental validations are described in detail by Yoo et al. (2015). It is noted that, in the present study, an additional layer of the polyimide tape was attached to the ITO film ( $700 \mathrm{~nm}$ thick) as shown in Figure 4; the specific purpose of using this tape is described later in section 2.3. Since the ITO film is opaque to the IR spectral range of our interest (i.e., 3-5 $\mu$ m), any IR radiation from the polyimide film surface touching the fluid will not reach the IR camera. In addition, despite the thinness of the polyimide tape (26 $\mu \mathrm{m}$ thick), the temperature difference between the ITO film and the inner surface of polyimide tape touching the fluid cannot simply be disregarded, especially in the high heat flux condition because the thermal conductivity of the elements composing the polyimide tape is low (i.e., polyimide film: $0.12 \mathrm{~W} / \mathrm{m}-\mathrm{K}$; silicone adhesive: $0.2 \mathrm{~W} / \mathrm{m}-\mathrm{K})$. Accordingly, we estimated the inner surface temperature of the polyimide film touching the fluid by solving the steady-state heat conduction equation (1D) based on the time-averaged ITO surface temperature and surface heat flux obtained by the algorithm described in Yoo et al. (2015). Then, this inner surface temperature, i.e., wall temperature was used to evaluate the wall heat transfer coefficient and Jacob number (Ja) during this study.

\subsection{Single nucleation site approach}

We controlled the number of nucleation sites (1) and its location during this study by creating a preferential single nucleation site at the specified axial location, i.e., $\mathrm{L} / \mathrm{L}_{0} \approx 0.41$ (see Figure 2). For this purpose, a thin polyimide tape with a silicone adhesive (CAPLINQ Corp.) with a total thickness of $26 \mu \mathrm{m}$ (polyimide film: $13 \mu \mathrm{m}$, silicone adhesive: $13 \mu \mathrm{m}$ ) was attached on the ITO-coated glass surface (see Figure 4), and a small cavity was created on top 
of the polyimide film using a sharp needle. The surface of the polyimide film attached was smooth except for the cavity region; the surface roughness was estimated to be about $3 \mathrm{~nm}$ as measured by Atomic Force Microscopy. After the degassing process, to activate the nucleation on the single artificial cavity, sufficient power was initially supplied to the heater under low liquid flow condition until boiling initiated at numerous sites including the intended one. Due to the complete degassing of the fluid as well as the smooth wall surface condition, such inception of vigorous boiling normally required very high heater power. After activating numerous nucleation sites over the entire heated area, the heater power was decreased again until only the nucleation site located on the artificial cavity remained activated. Then, while maintaining the single nucleation site, test conditions such as liquid mass flux and wall heat flux were adjusted until reaching the targeted test condition. This process was facilitated due to the preferential nucleation on the single artificial cavity compared to the other regions within the heated area. Thus, we always kept the single nucleation site in this manner at the same location $\left(\mathrm{L}_{\mathrm{L}} \approx 0.41\right)$, and the boiling behaviors originating from the same cavity were investigated under various subcooled flow boiling conditions.

\subsection{Enhanced effort on image (or data) acquisition and analysis}

Due to the strong stochastic nature of boiling, a sufficient amount of experimental data is required to correctly characterize the complex features of subcooled boiling flow at a given test condition. That is, the amount of experimental data must be large enough to ensure reliable statistics of the measured parameters. In the present work, HSC 1 and HSC 3 took 36,000 and 40,000 consecutive images, respectively, for each test case. As mentioned, the recording speed applied was $10 \mathrm{kHz}$ for HSC 1 and $0.5 \mathrm{kHz}$ for HSC 3, and thus the measurement period for HSC 1 and HSC 3 was 3.6 s and 80 s, respectively. From HSC 2, about 12,000 consecutive images were taken at a recording speed of $1 \mathrm{kHz}$, which were primarily used to qualitatively describe bubble motions downstream of the test section.

In order to analyze the bubbles' behavior, all the images acquired were first converted to binary images via image processing; the efficient image processing algorithm to address the numerous images of nucleating/departing bubbles for the side view measurement such as HSC 1 is described in Yoo et al. (2014). Also, the processed images of bubbles were analyzed automatically using the macro function of image analysis software. Specifically, the size of bubbles, their positions (i.e., center of mass location) and displacement between the consecutive image pairs, etc. were evaluated using ImageJ (Rasband, 1997-2014) and its plugin software MTrack2 (Stuurman, 2003). During this 
process, the poor quality of processed bubble images were filtered out by applying a certain criterion of bubble shape (e.g., circularity $\geq 0.65$ ), which was to keep the reliability of the present analysis. This criterion was determined based on the preliminary tests with various deformed bubble images to confirm if it is proper to remove only the bad quality images (e.g., broken bubbles, background noises) produced while converting the original images to binary ones. Also, to investigate the axial development of bubble size or bubble velocity within test section, the axial flow path viewed from HSCs was first partitioned into 200 400 sectors for HSC 1 and 100 200 sectors for HSC 3. Then, the bubbles captured within each sector during the measurement period were analyzed to obtain the average bubble size or bubble velocity at different axial locations of test section. Largely, more than 300-500 bubble samples were captured within each sector during the measurement period (3.6 s) of HSC 1 while $10^{3}-10^{4}$ order of bubbles were captured within each sector during the measurement period of HSC 3 (80 s).

For the measurement of wall temperature and wall heat flux, a total of 2,000 2-D thermal images $(1000 \times 34$ pixels) covering the entire heated area were recorded for each boiling test condition at a frame rate of $100 \mathrm{~Hz}$ using an IR camera. The raw data from the thermal images were processed by applying the calibration algorithm (Yoo et al., 2015) and using the steady-state heat conduction equation, from which 2-D field of actual wall temperature and heat flux were obtained. However, it is noted that only 1,700 out of the 2,000 processed data (2-D) were finally used to evaluate the time-averaged values (e.g., wall heat transfer coefficient) because the calibration algorithm requires the initial decay time until it tracks the true value (Yoo et al., 2015).

Figures 5 and 6 summarized the automatic image analyses and data processing procedures for the measurements from HSC 1, HSC 3 and IR camera, in which specific measurement parameters available from each camera are also presented. Note, however, that during this study HSC 2 was used only to qualitatively describe the bubbles motion downstream in the test channel (e.g., sliding or lift-off, etc.), and thus an image analysis for quantitative analysis was not conducted. The bubble and wall heat transfer parameters that can be measured from the present experimental setup are quite extensive, including (i) nucleating/departing bubbles behavior at a nucleation site, (ii) bubble size and growth behavior along the flow path, (iii) axial bubble velocity, (iv) bubbles release frequency, (v) bubbles' coalescence while sliding, (vi) bubble size distribution and its axial development, (vii) timeaveraged wall heat transfer coefficient, etc. In this first part of two companion papers, however, rather than directly discussing the experimental results, we first investigate the level of quality of experimental data produced based on the present experimental and analysis strategy. Specifically, the reliability and associated uncertainties of quantified 
experimental results obtained from the measurements of HSC 1, HSC 3 and IR thermometry is discussed in detail in the following section.

\section{Data quality verification}

\subsection{Repeatability test}

After the image (or data) acquisition and analyses were performed as described in section 2.4, the measurements reliability was evaluated in terms of statistical significance. To this end, a repeatability test was conducted for one of the subcooled flow boiling experimental conditions. The repeatability test results are discussed in this section, which provides crucial implications to evaluate the data quality obtained from the present work.

As mentioned in section 2.4, the size of the bubbles and their growth along the flow path is one of the main interests of this study. Figure 7 presents the repeatability test results on the axial variation of average bubble size near the single nucleation site $\left(0.41<\mathrm{L} / \mathrm{L}_{0}<0.45\right)$; the results for the measurements from HSC 1 are shown on the left side of Figure 7 while those for the measurements from HSC 3 are shown on the right side. The repeatability tests 1 and 2 were performed at different times under identical test conditions. Due to the different spatial and temporal resolutions between HSC 1 and HSC 3, we can see that the level of detail that can be captured for the bubble growth behavior within the same region is quite different. At present, however, we only focus on the repeatability issue associated with the two different image acquisition methods of HSC 1 and HSC 3.

The left side of Figure 7 shows the overall bubble growth behavior along the flow path observed by HSC 1, in which the two test results (repeatability tests 1 and 2) look largely repeatable. However, the bubble size shows noticeable differences locally, up to $\sim 0.2 \mathrm{~mm}$ at the axial location $0.41<\mathrm{L} / \mathrm{L}_{0}<0.415$. It was found that such differences occurred at the region where fluctuating behaviors of bubble size were enhanced due to the coalescence and bouncing motion of bubbles near the nucleation site (more details about this will be discussed in Part 2 of this study). As for this, the differences between the two tests shown in Figure 7 (left) could be due to one or more of the following issues: First, the sample size used for the statistical average might have not been insufficient. Second, the measurement period of $3.6 \mathrm{~s}$ for HSC 1 might not be long enough to characterize the bubble size variation resulting from the random (stochastic) nature of boiling. Third, the characteristics of heated surface or artificial cavity might have changed during the time between the two repeatability tests. Fourth, the uncertainty of the test boundary conditions, e.g., liquid flow rate and wall heat flux, may have affected the boiling process. 
Of the four issues listed, the authors regard the first and second as the dominant reasons for the discrepancy shown on the left side of Figure 7; and this argument can be supported by the improved repeatability results obtained by HSC 3 shown on the right side of Figure 7. As discussed, HSC 3 measured bubble motions along the flow path with a lower recording speed $(0.5 \mathrm{kHz})$ compared to that of HSC $1(10 \mathrm{kHz})$. Meanwhile, the measurements by HSC 3 were performed over $80 \mathrm{~s}$ while the measurement period of HSC 1 was only $3.6 \mathrm{sec}$. Although the total number of images taken by HSC 1 and HSC 3 were comparable, these different recording specifics can make a significant difference in the quality of the resulting statistics. This is because the bubbles were generated and moved much less within the relatively short measurement time of HSC 1. As a result, the number of bubbles (or samples) captured during the measurement period was relatively limited for HSC 1 (300-500 bubbles) compared to that for HSC 3 ( $10^{3}-10^{4}$ order of bubbles). Necessarily, the uncertainty of the statistics obtained based on the measurements of HSC 1 increase due to the smaller sample size and/or limited measurement time, which is attributed to the inherent stochastic nature (randomness) of data taken during the flow boiling process. Mathematically, Eq. (1) explains why the average bubble size based on the measurement of HSC 3 can induce better repeatability (or less uncertainty introduced by the randomness of measured data) than those of HSC 1:

$$
\delta \mathrm{s}(\bar{x})=\sigma_{x} / \sqrt{N}
$$

where $x$ denotes a random variable, $\bar{x}$ is the statistical average, $\delta s(\bar{x})$ is the standard uncertainty of $\bar{x}, \sigma_{x}$ is the standard deviation of $x$, and $N$ is the number of samples.

Obviously, the uncertainty defined in Eq. (1) will increase as the statistical variation of measured data increases or as the available number of samples $N$ decreases. Thus, it is important to take this into account as a part of experimental uncertainty, especially when the sample size is limited relative to the statistical variation of measured data like in the measurements from HSC 1; the details about the estimated uncertainty values will be described in section 3.2. In addition, Figure 7 (left) implies that the sample size and/or the measurement time of HSC 1 was not enough to properly characterize the statistics of bubble size at the local region $0.41<\mathrm{L} / \mathrm{L}_{0}<0.415$ under the given test condition despite the numerous images analyzed (36,000 images, see section 2.4); for this reason, the maximum discrepancy of average bubble size between the two repeatability tests is observed larger than the uncertainty suggested in section 3.2. Nevertheless, it should be noted that the experimental condition shown in Figure 7 caused an exceptionally large fluctuation of bubble size (i.e., large $\sigma_{x}$ ) due to bouncing bubbles at this near nucleation site region $\left(0.41<\mathrm{L} / \mathrm{L}_{0}<0.415\right)$ among the tests during this study, and the sample number was relatively 
limited due to the low frequency of bubble release from the nucleation site. Therefore, according to Eq. (1), a lower uncertainty (or better repeatability) is expected for the other tests at different conditions. Also, this issue hardly affected the quality of other measurement by HSC 1 such as bubble velocity as is discussed below.

In Figure 8, the repeatability of axial bubble velocity measured by HSC 1 is presented, which shows good agreement between repeatability tests 1 and 2 within the region $0.41<\mathrm{L} / \mathrm{L}_{0}<0.45$. It is interesting to note that the repeatability of axial bubble velocity is observed to be better than that of bubble size measured by HSC 1 within the same region (see Figure 7 - left). This implies that the local fluctuation of bubble size which caused a difference in average bubble size between the two repeatability tests barely affected the axial bubble velocity.

In Figure 9, presented are the repeatability test results for the three bubble parameters measured by HSC 3 over the longer flow path $0.41<\mathrm{L} / \mathrm{L}_{0}<0.75$. Specifically, the bubble size, the axial bubble velocity and the two bubble size distribution measured at $\mathrm{L} / \mathrm{L}_{0}=0.43$ and 0.71 , respectively. As discussed previously, the statistical average uncertainty depends on the sample size and the inherent randomness (i.e., stochastic feature) of the measured parameters at a given boiling condition. The excellent agreement between the repeated measurements shown in Figure 9 implies that the bubble characteristics are well-represented by the current image acquisition strategy using HSC 3. In particular, it is interesting to find that the bubble size distribution shown on the bottom right of Figure 9 deviates slightly from a normal distribution due to the increasing portion of smaller bubbles downstream of the test section (i.e., $\mathrm{L} / \mathrm{L}_{0}=0.71$ ). This is due to the fact that bubbles often lifted off from the wall after sliding a short distance and were subsequently propelled into the bulk subcooled liquid while condensing at the downstream region. The probabilistic density function shown on the bottom right of Figure 9 indicates that even such characteristic of bubble behavior is well captured. From this, we can conclude that the sample size taken from the measurement of HSC 3 was large enough to ensure reliable statistics of various measured bubble parameters. Also, it can be expected that the uncertainty introduced by the randomness (i.e., stochastic feature) of the measured data be negligibly small.

Figure 10 shows a result of repeatability test on the time-averaged wall temperature measured by IR thermometry within the whole heated flow path $0 \leq \mathrm{L} / \mathrm{L}_{0} \leq 1$. This figure clearly shows how dramatically wall heat transfer at the wall touching the fluid increased due to the presence of a single nucleation site at axial location $\mathrm{L} / \mathrm{L}_{0} \approx 0.41$. In addition, excellent agreement is observed between the repeatability tests 1 and 2 in both single phase region $\left(0<\mathrm{L} / \mathrm{L}_{0}<0.41\right)$ and bubble-affected two-phase flow region $\left(0.41 \leq \mathrm{L} / \mathrm{L}_{0} \leq 1\right)$. This indicates that the time- 
averaged wall heat transfer characteristics affected by boiling bubbles can also be represented well by the current strategy of thermal image acquisition described in section 2.4.

\subsection{Uncertainty analysis}

This section discusses the uncertainty quantification for the bubble and wall heat transfer measurements during the present work. Table 1summarizes the combined uncertainties for the measured parameters from each camera as well as the specific uncertainty sources contributing to them $\left(\mathrm{D}_{\mathrm{b}}\right.$ and $\mathrm{u}$ denote the bubble size and sliding bubble velocity, respectively.). The detailed process and associated discussion is given as below.

For bubble size measurement using HSC 1, several sources of uncertainty exist due to (i) camera spatial resolution, (ii) limited sample size relative to inherent randomness (stochastic nature) of measured data, (iii) userdependent image processing algorithm, etc. Among them, due to the high resolution and quality of bubble images achieved by HSC 1 (see Figures 3 and 5), the major source of uncertainty is expected to be the limited sample size due to the limited measurement time of HSC 1 (3.6 s) as discussed in section 3.1. This uncertainty can be estimated by Eq. (1). However, we should note that the magnitude of such uncertainty depends on experimental conditions and on the location where the bubbles are captured within the test section. Instead of addressing each of them separately, we estimated the uncertainty in a conservative manner by evaluating the uncertainties at regions where $\sigma_{x}$ was significantly larger for each experimental condition. In general, within the measurement view of HSC 1 (i.e., $0.41<\mathrm{L} / \mathrm{L}_{0}<0.45$ ), the fluctuations of bubble size were observed to become significantly enhanced at the regions where the bubbles detached from the wall and reattached again (i.e., bouncing motion) near the single nucleation site. Or, if such fluctuation of bubble size due to the bouncing motion were insignificant given the experimental conditions, the $\sigma_{x}$ tended to be slightly larger as the bubbles grew while sliding downstream. Thus, we analyzed the bubble size distribution at such regions over the entire experimental set and estimated the uncertainties using Eq. (1). Then, the maximum value was selected as the uncertainty introduced by the limited sample size or the stochastic nature of measured data (bubble size). The uncertainty obtained in this way was $\pm 14.9 \mu \mathrm{m}$. Combining this with the uncertainty introduced by the spatial resolution of HSC 1 (Kline and McClintock, 1953), the total uncertainty for the bubble size measurements by HSC 1 was determined to be $\pm 17.5 \mu \mathrm{m}$.

Similarly, for the uncertainty of axial bubble velocity measured by HSC 1, we analyzed the axial bubble velocity distribution at axial locations near the nucleation site $\left(\mathrm{L} / \mathrm{L}_{0} \approx 0.41\right)$ and a region further downstream 
$\left(\mathrm{L} / \mathrm{L}_{0} \approx 0.44\right)$ for the entire set of experiments. Then, using Eq. (1) the related uncertainties were estimated, from which the maximum value, $\pm 3.2 \times 10^{-3} \mathrm{~m} / \mathrm{s}$, was determined as the uncertainty for the axial bubble velocity measured by HSC 1. The uncertainties due to the erroneous detection of bubble positions (displacement) and due to the time interval between the image pairs were assumed negligible for HCS 1.

In contrast to the measurement of HSC 1, the sample size-induced uncertainty (i.e., number of bubbles) was not a major source of uncertainty for the bubble size measurement by HSC 3. This is due to the larger sample size and the longer period of measurement time (80 s) compared to that of HSC 1 (3.6 s) as discussed in section 2.4 . Instead, the limited spatial resolution used by HSC $3(78.7 \mu \mathrm{m})$ and the random errors introduced by the userdependent image processing algorithm were the most important sources of uncertainty. Thus, by combining such sources of uncertainty (Kline and McClintock, 1953), the uncertainty of bubble size measurement by HSC 3 was determined to be $\pm 153.9 \mu \mathrm{m}$. Also, in a similar context with the previous discussion, the uncertainty due to the limited sample size, which was discussed in section 3.1, was ignored for the uncertainty estimation of axial bubble velocity measured by HSC 3. Instead, due to the limited spatial resolution of HSC 3, the final uncertainty was estimated by considering the uncertainty of bubble positions used for the computation of the axial bubble velocity. This uncertainty can be calculated by the following relation:

$$
\delta s(u)=\sqrt{\left(\frac{\delta s\left(x_{i-1}\right)}{\Delta t}\right)^{2}+\left(\frac{\delta s\left(x_{i}\right)}{\Delta t}\right)^{2}}=\sqrt{2\left(\frac{\delta s(\Delta x)}{\Delta t}\right)}
$$

where $\delta \mathrm{s}(\mathrm{u})$ is the uncertainty of axial bubble velocity, $\delta \mathrm{s}\left(\mathrm{x}_{\mathrm{i}}\right)$ is the uncertainty of bubble position at $\mathrm{i}^{\text {th }}$ frame, $\Delta \mathrm{t}$ is the time interval between consecutive image pairs taken by HSC (i-1 and $\mathrm{i}$ are separated by a time interval $\Delta \mathrm{t}$ ), $\Delta \mathrm{x}$ is the displacement of a bubble during $\Delta \mathrm{t}$ and $\delta \mathrm{s}(\Delta \mathrm{x})$ is the uncertainty of bubble displacement. It is noted that for HSC 3 the uncertainty of a bubble position at each frame, i.e., $\delta \mathrm{s}\left(\mathrm{x}_{\mathrm{i}-1}\right.$ or $\left.\mathrm{x}_{\mathrm{i}}\right)$ in Eq. (2) was assumed to be half of the spatial resolution.

As a result, Eq. (2) resulted in the uncertainty of axial bubble velocity for HSC 3 as $\pm 2.8 \times 10^{-2} \mathrm{~m} / \mathrm{s}$. Also, it is noted that the "high-speed" measurement does not always guarantee a reliable velocity measurement because the uncertainty estimated by Eq. (2) increases as $\Delta \mathrm{t}$ decreases at a given spatial resolution of camera.

The uncertainty analysis for the temperature measurement of the heating surface (i.e., ITO film) through the same design of multi-layer wall was performed in our previous study (Yoo et al., 2015). However, during the present work an additional polyimide tape was attached to the ITO film as described in section 2.2. Thus, the uncertainty associated with the thermal properties of the elements composing the polyimide tape was additionally 
considered, i.e., $\pm 0.01 \mathrm{~W} / \mathrm{m}-\mathrm{K}$ was used as the uncertainty for the thermal conductivity of the polyimide film and the silicone adhesive. Consequently, the uncertainty for the wall temperature measurement was determined to be $0.77^{\circ} \mathrm{C}$. The experimental uncertainty for the heat flux measurement was estimated as $0.6 \mathrm{~kW} / \mathrm{m}^{2}$.

For the other measurements, the uncertainties of liquid flow rate, fluid temperature, and heater power given by the DC power supply were obtained as $\pm 0.05 \%$ of reading, $\pm 0.5^{\circ} \mathrm{C}$, and $\pm 1.1 \%$ (full scale), respectively.

\section{Summary and conclusions}

A novel experimental method with image acquisition/analysis strategy ensuring reliable measurement of various flow boiling parameters is discussed. For this study, a subcooled flow boiling experiment was conducted in a square, vertical, upward flow channel with a one-sided heater. To overcome the general difficulties of boiling parameter measurement through optical techniques, a single nucleation site was purposely activated through the entire heated area. This significantly reduced the phenomenological complexity resulting from the random presence of nucleation sites as well as the difficulty of visual observation.

The bubble and wall heat transfer parameters were measured with three high-speed cameras and one IR camera; the vapor bubbles were observed using both micro- and macroscopic observation views while the wall temperature was measured with a viewing scale covering the whole heater. This measurement strategy allowed simultaneously observing the various aspects of bubble characteristics with multiple scales (both space and time) as well as their impact on wall heat transfer.

In addition, due to the high quality of bubble images, numerous images obtained from the high-speed cameras were analyzed with high fidelity by applying an automatic image processing algorithm. This led to an excellent repeatability and thus reliability of statistics obtained from the present data. Also, the major sources of uncertainty, including the uncertainty due to the randomness of measured data, are discussed which revealed that the uncertainties and their sources depend substantially on the camera resolutions applied.

It is important to note that, until recently most of the flow boiling studies in the literature have little argued about the quality of experimental data from the perspectives discussed in this paper. Besides, the efforts to quantify uncertainties related to such limitations of visualization techniques are scarce. In this respect, the present study clearly shows the important issues to consider in order to achieve truly reliable boiling data; and this will be 
especially crucial when efforts are made to develop and validate correlations/models based on the measured data from any boiling experiments.

The specific physical insight obtained from the present experimental work will be discussed in Part 2 of this study.

\section{Acknowledgment}

The authors acknowledge the support by CASL (Consortium for Advanced Simulation of Light Water Reactors) a DOE Energy Innovation Hub for this research. The support is gratefully acknowledged. 


\section{List of Figures}

Figure 1. Subcooled flow boiling loop with a vertical square test section

Figure 2. Optical measurement of vapor bubbles and wall temperature in the subcooled flow boiling experiment

Figure 3. Representative bubble images taken from the three high-speed cameras

Figure 4. Detailed heater wall design and wall temperature measurement using IR thermometry (Not to scale)

Figure 5. Image analysis procedure and measured parameters from HSC 1

Figure 6. Image analysis procedure and measured parameters from HSC 3 and IR thermometry

Figure 7. Repeatability test on the bubble size based on the measurements of HSC 1 (left) and HSC 3 (right)

Figure 8. Repeatability test on the axial bubble velocity based on the measurement of HSC 1

Figure 9. Repeatability test on the bubble size (upper left), axial bubble velocity (upper right) and statistical

distributions of bubble size (at $\mathrm{L} / \mathrm{L}_{0}=0.43$, bottom left; at $\mathrm{L} / \mathrm{L}_{0}=0.71$, bottom right) measured by HSC 3

Figure 10. Repeatability test on the wall temperature obtained by IR thermometry 


\section{List of Tables}

Table 1. Uncertainty sources of measured data from each camera 


\section{References}

Abdelmessih, A.H., Nangia, S., Hooper, F.C., 1972. Flow effects on bubble growth and collapse in surface boiling. Int J Heat Mass Tran 15, 115-118.

Anglart, H., Nylund, O., Kurul, N., Podowski, M.Z., 1997. CFD prediction of flow and phase distribution in fuel assemblies with spacers. Nucl Eng Des 177, 215-228.

Bae, B.U., Yun, B.J., Yoon, H.Y., Song, C.H., Park, G.C., 2010. Analysis of subcooled boiling flow with one-group interfacial area transport equation and bubble lift-off model. Nucl Eng Des 240, 2281-2294.

Chu, I.C., No, H.C., Song, C.H., 2011. Bubble lift-off diameter and nucleation frequency in vertical subcooled boiling flow. J Nucl Sci Technol 48, 936-949.

Ivey, H.J., 1967. Relationships between bubble frequency departure diameter and rise velocity in nucleate boiling. Int J Heat Mass Tran 10, 1023-1040.

Klausner, J.F., Mei, R., Bernhard, D.M., Zeng, L.Z., 1993. Vapor bubble departure in forced-convection boiling. Int J Heat Mass Tran 36, 651-662.

Kline, S.J., McClintock, F.A., 1953. Describing uncertainties in single-sample experiments. Mechanical Engineering 75, 3-8.

Kocamustafaogullari, G., Ishii, M., 1983. Interfacial area and nucleation site density in boiling systems. Int J Heat Mass Tran 26, 1377-1387.

Krepper, E., Rzehak, R., 2011. CFD for subcooled flow boiling: Simulation of DEBORA experiments. Nucl Eng Des 241, 3851-3866.

Li, S.D., Tan, S.C., Xu, C., Gao, P.Z., Sun, L.C., 2013. An experimental study of bubble sliding characteristics in narrow channel. Int J Heat Mass Tran 57, 89-99.

Maurus, R., Sattelmayer, T., 2006. Bubble and boundary layer behaviour in subcooled flow boiling. Int J Therm Sci 45, 257-268.

Okawa, T., Ishida, T., Kataoka, I., Mori, M., 2005. On the rise paths of single vapor bubbles after the departure from nucleation sites in subcooled upflow boiling. Int J Heat Mass Tran 48, 4446-4459.

Podowski, M.Z., Podowski, R.M., 2009. Mechanistic multidimensional modeling of forced convection boiling heat transfer. Sci Technol Nucl Ins. 
Prodanovic, V., Fraser, D., Salcudean, M., 2002. Bubble behavior in subcooled flow boiling of water at low pressures and low flow rates. Int J Multiphas Flow 28, 1-19.

Rasband, W.S., 1997-2014. ImageJ. http://imagej.nih.gov/ij/, U.S National Institutes of Health, Bethesda, Maryland, USA.

Situ, R., Hibiki, T., Ishii, M., Mori, M., 2005. Bubble lift-off size in forced convective subcooled boiling flow. Int J Heat Mass Tran 48, 5536-5548.

Situ, R., Ishii, M., Hibiki, T., Tu, J.Y., Yeoh, G.H., Mori, M., 2008. Bubble departure frequency in forced convective subcooled boiling flow. Int J Heat Mass Tran 51, 6268-6282.

Stuurman, N., 2003. MTrack2. http://valelab.ucsf.edu/ nstuurman/ijplugins/MTrack2.html.

Thorncroft, G.E., Klausner, J.F., Mei, R., 1998. An experimental investigation of bubble growth and detachment in vertical upflow and downflow boiling. Int J Heat Mass Tran 41, 3857-3871.

Unal, H.C., 1976. Maximum bubble diameter, maximum bubble growth time and bubble growth rate during subcooled nucleate flow boiling of water up to 17.7 MN/M2. Int J Heat Mass Tran 19, 643-649.

Yeoh, G.H., Cheung, S.C.P., Tu, J.Y., Ho, M.K.M., 2008. Fundamental consideration of wall heat partition of vertical subcooled boiling flows. Int J Heat Mass Tran 51, 3840-3853.

Yoo, J., Estrada-Perez, C.E., Hassan, Y.A., 2014. A proper observation and characterization of wall nucleation phenomena in a forced convective boiling system. Int J Heat Mass Tran 76, 568-584.

Yoo, J., Estrada-Perez, C.E., Hassan, Y.A., 2015. An accurate wall temperature measurement using infrared thermometry with enhanced two-phase flow visualization in a convective boiling system. Int J Therm Sci 90, 248266.

Zeng, L.Z., Klausner, J.F., Bernhard, D.M., Mei, R., 1993. A unified model for the prediction of bubble detachment diameters in boiling systems: 2. Flow boiling. Int J Heat Mass Tran 36, 2271-2279. 
Degassing tank

Separator

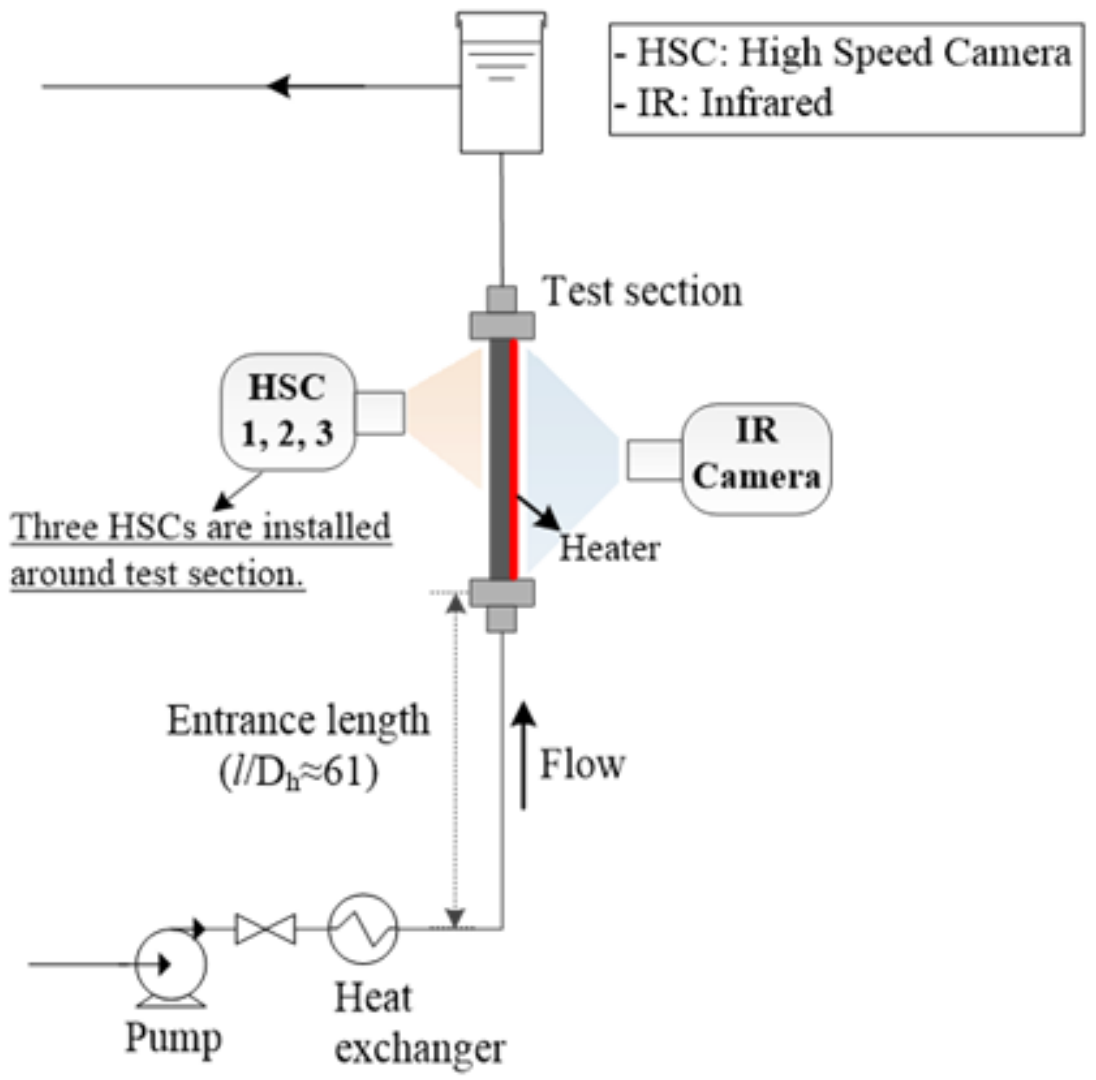

Figure 1. Subcooled flow boiling loop with a vertical square test section 


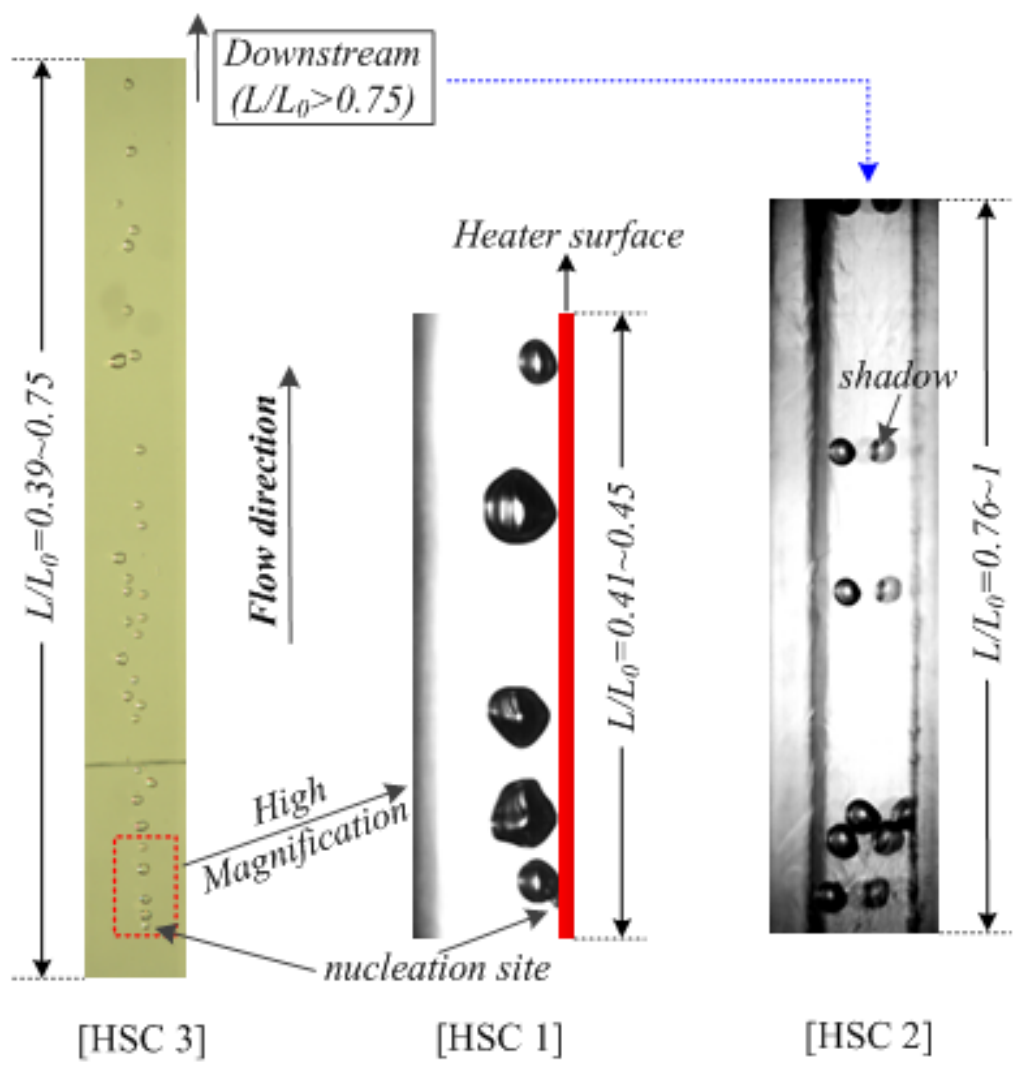

Figure 3. Representative bubble images taken from the three high-speed cameras 


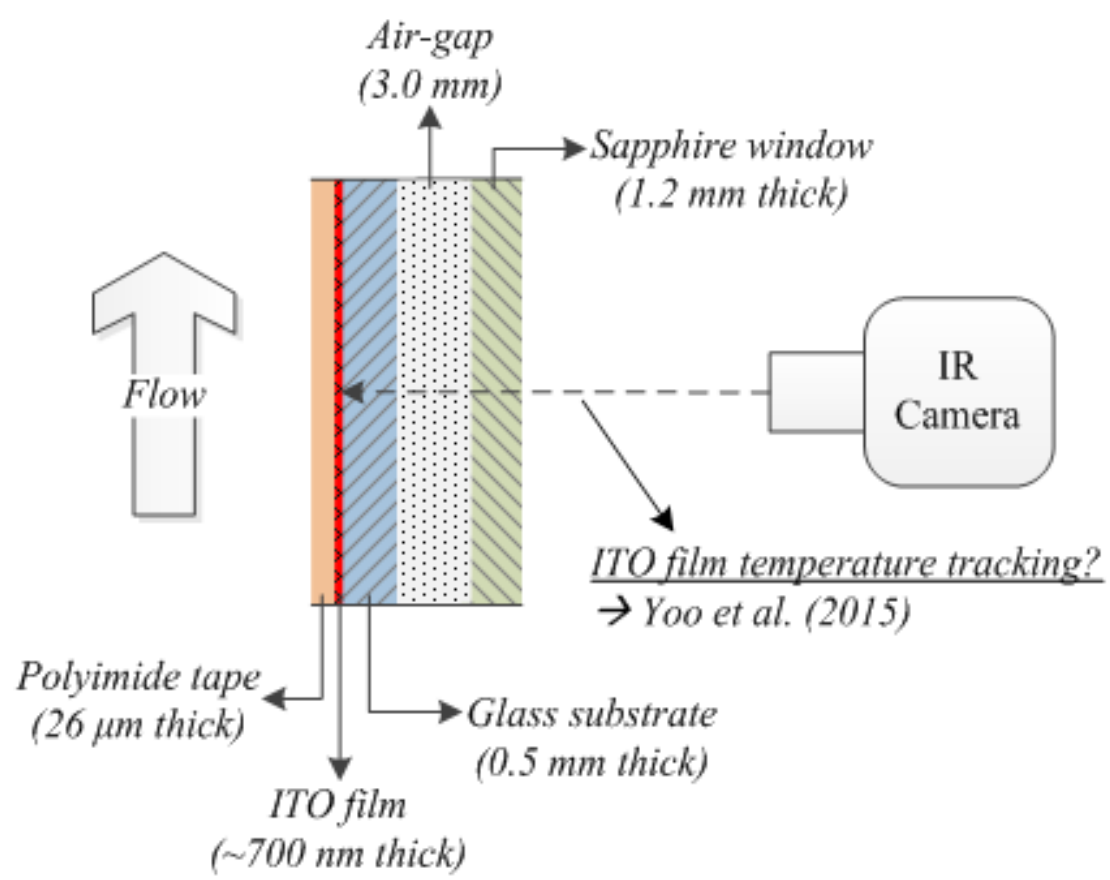

Figure 4. Detailed heater wall design and wall temperature measurement using IR thermometry (Not to scale) 
Image processing (Yoo et al., 2014)

(convert to binary images)

Single mucleation site

$\left(L / L_{\theta} \approx 0.41\right)$

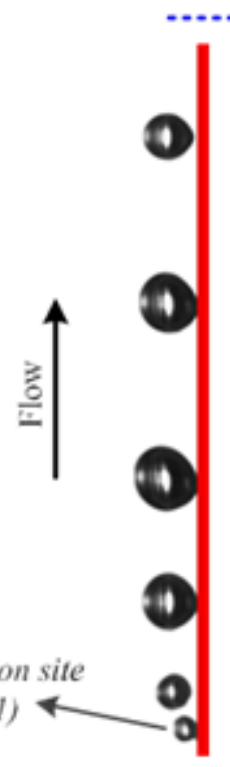

[Original image]

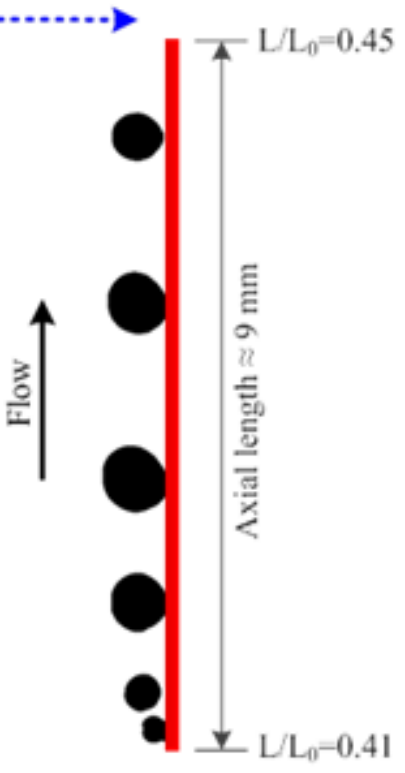

[Processed image]
36,000 consecutive images were analyzed at each test condition (using macro function of ImageJ)

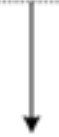

\section{Measured parameters}

1. Bubbles mucleating/departure behavior

2. Bubbles growth behavior

3. Bubbles release frequency

4. Bubbles velocity

5. Bubbles coalescence (or bubble number variation)

\section{Visualization of Bubbles from HSC 1}

Figure 5. Image analysis procedure and measured parameters from HSC 1 

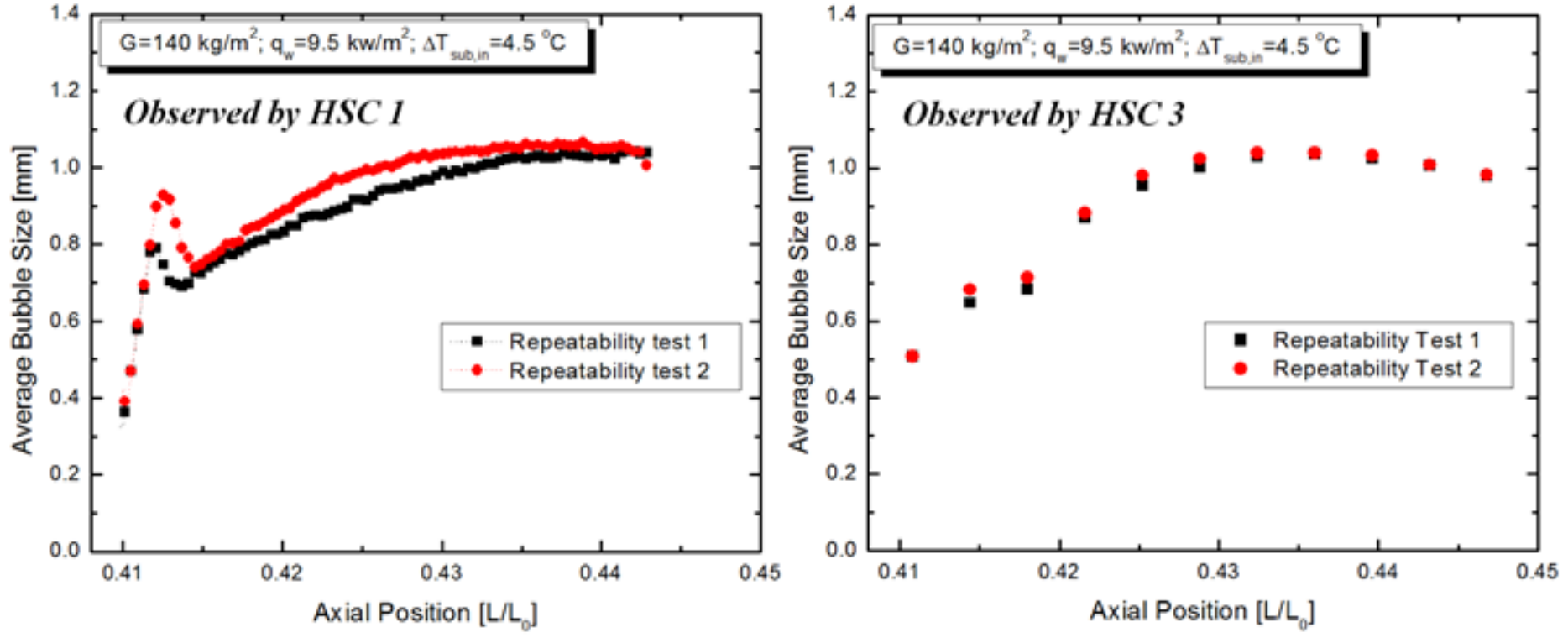

Figure 7. Repeatability test on the bubble size based on the measurements of HSC 1 (left) and HSC 3 (right) 


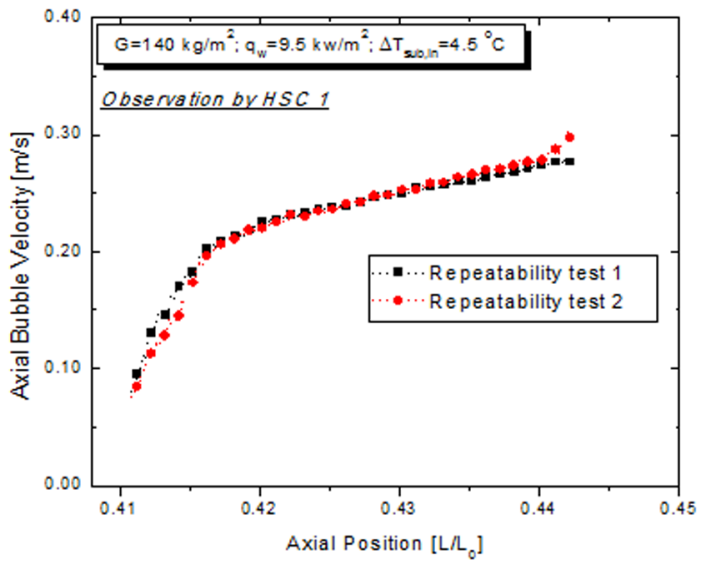

Figure 8. Repeatability test on the axial bubble velocity based on the measurement of HSC 1 

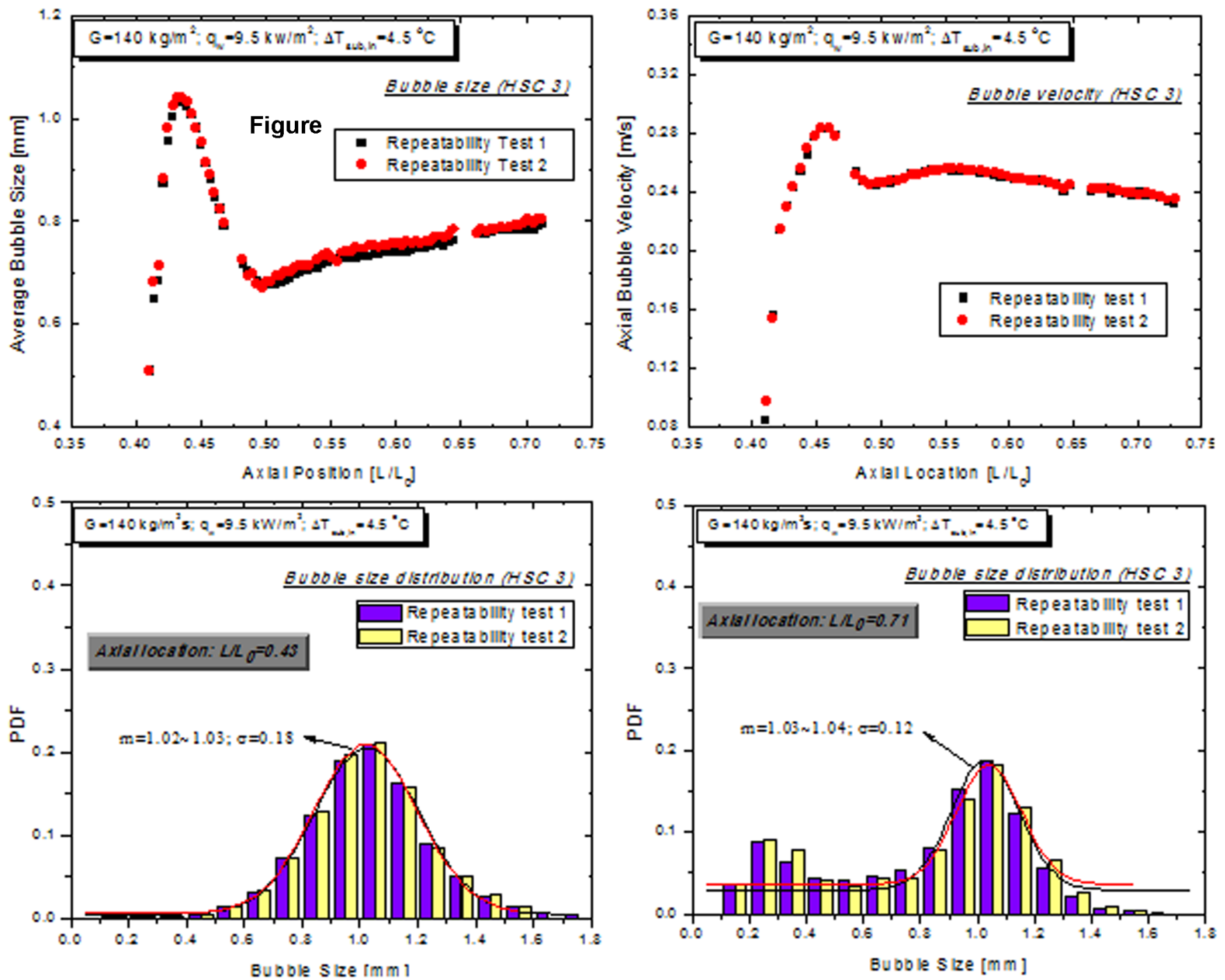

Figure 9. Repeatability test on the bubble size (upper left), axial bubble velocity (upper right) and statistical distributions of bubble size (at $\mathrm{L} / \mathrm{L}_{0}=0.43$, bottomleft; at $\mathrm{L} / \mathrm{L}=0.71$, bottom right) measured by HSC 3 


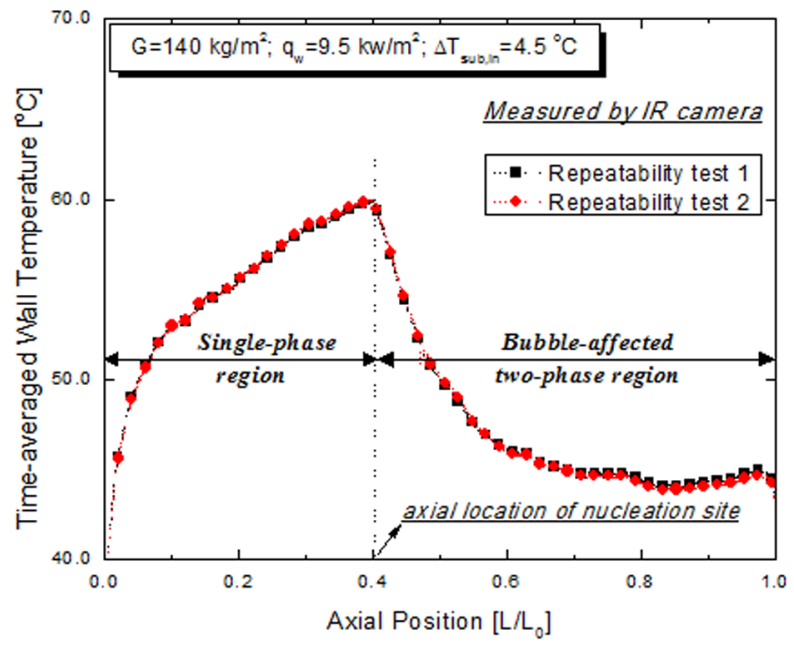

Figure 10. Repeatability test on the wall temperature obtained by IR thermometry 
Table 1. Uncertainty sources of measured data from each camera

\begin{tabular}{|c|c|c|c|c|c|}
\hline \multirow[t]{2}{*}{ Uncertainty source } & \multicolumn{2}{|c|}{$\begin{array}{c}\text { HSC } 1 \\
\text { (high resolution) }\end{array}$} & \multicolumn{2}{|c|}{$\begin{array}{c}\text { HSC } 3 \\
\text { (low resolution) }\end{array}$} & \multirow[t]{2}{*}{ IR camera } \\
\hline & $\delta \mathrm{s}\left(\mathrm{D}_{\mathrm{b}}\right)$ & $\delta \mathrm{s}(\mathrm{u})$ & $\delta \mathrm{s}\left(\mathrm{D}_{\mathrm{b}}\right)$ & $\delta \mathrm{s}(\mathrm{u})$ & \\
\hline Camera resolution & $\pm 9.09 \mu \mathrm{m}$ & - & $\pm 78.7 \mu \mathrm{m}$ & - & \multirow{4}{*}{$\begin{array}{l}\text { The uncertainties for } \\
\text { thermal properties of } \\
\text { heater wall materials } \\
\text { are considered as } \\
\text { described in Yoo et al., } \\
2015 \text {. }\end{array}$} \\
\hline $\begin{array}{l}\text { User-dependent } \\
\text { image processing }\end{array}$ & - & - & $\pm 132.3 \mu \mathrm{m}$ & - & \\
\hline Limited sample size & $\pm 14.9 \mu \mathrm{m}$ & $\pm 3.2 \times 10^{-3} \mathrm{~m} / \mathrm{s}$ & - & - & \\
\hline $\begin{array}{l}\text { Uncertainty of } \\
\text { bubble position }\end{array}$ & - & - & - & $\pm 2.8 \times 10^{-2} \mathrm{~m} / \mathrm{s}$ & \\
\hline $\begin{array}{l}\text { Combined } \\
\text { uncertainty }\end{array}$ & $\pm 17.5 \mu \mathrm{m}$ & $\pm 3.2 \times 10^{-3} \mathrm{~m} / \mathrm{s}$ & $\pm 153.9 \mu \mathrm{m}$ & $\pm 2.8 \times 10^{-2} \mathrm{~m} / \mathrm{s}$ & $\begin{array}{l}\text { Temperature: } \pm 0.77^{\circ} \mathrm{C} \\
\text { Heat flux: } \pm 0.6 \mathrm{~kW} / \mathrm{m}^{2}\end{array}$ \\
\hline
\end{tabular}

